\title{
Article
}

\section{Pseudo-valuations and pseudo-metric on JU-algebras}

\author{
Usman Ali ${ }^{1}$, Moin A. Ansari ${ }^{2}$ and Masood Ur Rehman ${ }^{3, *}$ \\ 1 Center for Advanced Studies in Pure and Applied Mathematics, Bahauddin Zakariya University, Multan, Pakistan.; \\ uali@bzu.edu.pk \\ 2 Department of Mathematics,college of Science, Post Box 2097, Jazan University, Jazan, KSA.; maanari@jazanu.edu.sa \\ 3 Department of Mathematics, Abbottabad University of Science and Technology, Abbottabad, Pakistan. \\ * Correspondence: masoodqau27@gmail.com
}

Received: 1 January 2019; Accepted: 19 November 2019; Published: 16 December 2019.

\begin{abstract}
In this paper we have introduced the concept of pseudo-valuations on JU-algebras and have investigated the relationship between pseudo-valuations and ideals of JU-algebras. Conditions for a real-valued function to be a pseudo-valuation on JU-algebras are given and results based on them have been shown. We have also defined and studied pseudo-metric on JU-algebras and have proved that $\vartheta$ being a valuation on a JU-algebras $A$, the operation $\diamond$ in $A$ is uniformly continuous.
\end{abstract}

Keywords: JU-algebra, JU-ideal, valuation, pseudo-valuations, pseudo metric.

MSC: 06F35, 03G25, $03 C 05$.

\section{Introduction}

$\mathbf{P}$ seudo-valuations in residuated lattices was introduced by Busneag [1] where many theorems based on pseudo-valuations in lattice terms and their extension theorem for residuated lattices to pseudo-valuation from valuations are shown using the model of Hilbert algebras [2]. But in fact Pseudo-valuations on a Hilbert algebras was initially introduced by Busneag [3] where it is proved that every pseudo-valuation induces a pseudometric on a Hilbert algebra. Further Busneag [2] proved many results on extensions of pseudo-valuation.

Logical algebras have become the keen interest for researchers in recent years and intensively studied under the influence of different mathematical concepts. Doh and Kang [4] introduced the concept of pseudo-valuation on BCK/BCI algebras and studied several results based on them. Ghorbani [5] defined congruence relations and gave quotient structure of BCI-algebras based on pseudo-valuation. Zhan and Jun [6] studied pseudo valuation on $R_{0}$-algebras. Based on the concept of pseudo-valuation in $R_{0}$-algebras, Yang and Xin [7] characterized pseudo pre-valuations on EQ-algebras. Mehrshad and Kouhestani studied Pseudo-Valuations on BCK-Algebras [8]. Pseudo-valuations on a BCC-algebra was introduced by Jun et al. [9], where they have shown that binary operation in BCC-algebras is uniformly continuous. Recently Moin et al. [16] introduced JU-algebras and their $p$-closure ideals.

UP-algebras were introduced by Iampan [10] as a new branch of logical algebras. Naveed et al. [11] introduced the concept of cubic KU-ideals of KU-algebras. Moin and Ali [12] have given the concept of roughness in KU-Algebras recently whereas rough set theory in UP-algebras have been introduced and studied by Moin et al. [13]. Next, graph associated to UP-algebras was introduced by Moin et al. [14]. Daniel studied pseudo-valuations on UP-algebras in [15].

In this paper, we focus on pseudo-valuation which is applied to JU-algebras and discuss related results. We define pseudo-valuations on JU-algebras using the model of Busneag and introduce a pseudo-metric on JU-algebras. We also prove that the binary operation defined on JU-algebras is uniformly continuous under the induce pseudo-metric.

\section{Preliminaries and basic properties of JU-algebras}

In this section, we shall introduce JU-algebras, JU-subalgebras, JU-ideals and other important terminologies with examples and some related results. 
Definition 1. An algebra $(A, \diamond, 1)$ of type $(2,0)$ with a single binary operation $\diamond$ is said to be JU-algebras satisfying the following identities: for any $u, v, w \in X$,

$$
\begin{aligned}
& \left(J U_{1}\right)(u \diamond v) \diamond[(v \diamond w) \diamond(u \diamond w)]=1, \\
& \left(J U_{2}\right) 1 \diamond u=u, \\
& \left(J U_{3}\right) u \diamond v=v \diamond u=1 \text { implies } u=v .
\end{aligned}
$$

We call the constant 1 of $X$ the fixed element of $X$. For the sake of convenience, we write $X$ instead of $(X, \diamond, 1)$ to represent a JU-algebra. We define a relation " $\leq "$ in $X$ by $v \leq u$ if and only if $u \diamond v=1$. If we add the condition $u \diamond 1=1$ for all $u \in X$ in the definition of JU-algebras, then we get that $X$ is a KU-algebra. Therefore, JU-algebra is a generalization of KU-algebras.

Lemma 2. If $X$ is a JU-algebra, then $(X, \leq)$ is a partial ordered set i.e.,
$\left(J_{4}\right) u \leq u$,
$\left(J_{5}\right) u \leq v, v \leq u$, implies $u=v$,
(J6) $u \leq w, w \leq v$, implies $u \leq v$.

Proof. Putting $v=w=1$ in $\left(J U_{1}\right)$ we get $u \diamond u=1$, i.e. $u \leq u$ which proves $\left(J_{4}\right)$. $\left(J_{5}\right)$ directly follows from $\left(J U_{3}\right)$. For $\left(J_{6}\right)$ take $u \leq w$ and $w \leq v$ implies that $w \diamond u=1$ and $v \diamond w=1$. By $\left(J U_{1}\right)$, we have $v \diamond u=1$ implies that $u \leq v$.

Further we have the following Lemma for a JU-algebra $X$.

Lemma 3. If $A$ is a JU-algebra, then following inequalities holds for any $u, v, w \in A$ :
$\left(J_{7}\right) u \leq v$ implies $v \diamond w \leq u \diamond w$,
$\left(J_{8}\right) u \leq v$ implies $w \diamond u \leq w \diamond v$,
$\left(J_{9}\right)(w \diamond u) \diamond(v \diamond u) \leq v \diamond w$,
$\left(J_{10}\right)(v \diamond u) \diamond u \leq v$.

Proof. $\left(J_{7}\right),\left(J_{8}\right)$ and $\left(J_{9}\right)$ follows from $\left(J U_{1}\right)$ by adequate replacement of elements. $\left(J_{10}\right)$ follows from $\left(J U_{1}\right)$ and Definition 1.

Next, we have the following Lemmas.

Lemma 4. Any JU-algebra $X$ satisfies following conditions for any $u, v, w \in A$,

$\left(J_{11}\right) u \diamond u=1$,

$\left(J_{12}\right) w \diamond(v \diamond u)=v \diamond(w \diamond u)$,

$\left(J_{13}\right)$ If $(u \diamond v) \diamond v=1$, then $A$ is a KU-algebra,

$\left(J_{14}\right)(v \diamond u) \diamond 1=(v \diamond 1) \diamond(u \diamond 1)$.

Proof. Putting $v=w=1$ in $J U_{1}$, we get; $u \diamond u=1$ which proves $\left(J_{11}\right)$. For $\left(J_{12}\right)$, we have $(w \diamond u) \diamond u \leq w$. By putting $v=1$ in $\left(J U_{1}\right)$ and using $\left(J_{7}\right)$, we get

$$
w \diamond(v \diamond u) \leq((w \diamond u) \diamond u) \diamond(v \diamond u) .
$$

Replace $w$ by $w \diamond u$ in $\left(J U_{1}\right)$, we get $[v \diamond(w \diamond u)] \diamond[((w \diamond u) \diamond u) \diamond(v \diamond u)]=1$, which implies

$$
((w \diamond u) \diamond u) \diamond(v \diamond u) \leq v \diamond(w \diamond u) .
$$

From (1), (2) and Lemma $2\left(J_{6}\right)$ we get,

$$
w \diamond(v \diamond u) \leq v \diamond(w \diamond u)
$$

Further by replacing $v$ with $w$ and $w$ with $v$ in (3), we get

$$
v \diamond(w \diamond u) \leq w \diamond(v \diamond u)
$$

Now (3), (4) and $\left(J_{5}\right)$ yields, $w \diamond(v \diamond u)=v \diamond(w \diamond u)$. 
In order to prove $\left(J_{13}\right)$, we just needs to show that $u \diamond 1=1, \forall u \in A$. Replacing $v \rightarrow 1, u \rightarrow 1, w \rightarrow u$ in $\left(J U_{1}\right)$, we obtained, $(1 \diamond u) \diamond[(u \diamond 1) \diamond(1 \diamond 1)]=1 \Rightarrow u \diamond[(u \diamond 1) \diamond 1]=1 \Rightarrow u \diamond 1=1$ (by using $v=1$ in the given condition of $\left.\left(J_{13}\right)\right)$.

Using $\left(J_{12}\right)$ for any $u, v \in A$ in the followings we see that, $(v \diamond 1) \diamond(u \diamond 1)=(v \diamond 1) \diamond u \diamond[(v \diamond u) \diamond(v \diamond u)]=$ $(v \diamond 1) \diamond[(v \diamond u) \diamond(u \diamond(v \diamond u))]=(v \diamond u) \diamond[(v \diamond 1) \diamond(v \diamond(u \diamond u))]=(v \diamond u) \diamond[(v \diamond 1) \diamond(v \diamond 1)]=(v \diamond u) \diamond 1$, which shows that $\left(J_{14}\right)$ holds.

Definition 5. A non-empty subset $I$ of a JU-algebra $A$ is called a JU-ideal of $A$ if it satisfies the following conditions:

(1) $1 \in I$,

(2) $u \diamond(v \diamond w) \in I, v \in I$ implies $u \diamond w \in I$, for all $u, v, w \in I$.

\section{Pseudo-valuations and pseudo-metric}

Definition 6. A real-valued function $\vartheta$ on a JU-algebra $A$ is called a pseudo-valuation on $A$ if it satisfies the following two conditions:

(1) $\vartheta(1)=0$,

(2) $\vartheta(u \diamond w) \leq \vartheta(u \diamond(v \diamond w))+\vartheta(v)$ for all $u, v, w \in A$.

A pseudo-valuation $\vartheta$ on a JU-algebra $A$ satisfying the following condition:

$\vartheta(u)=0 \Rightarrow u=1$ for all $u \in A$ is called a valuation on $A$.

Example 1. Let $A=\{1,2,3,4\}$ be a set with operation $\diamond$. A Cayley table for $A$ is defined as

\begin{tabular}{|l||l|l|l|l|}
\hline$\diamond$ & 1 & 2 & 3 & 4 \\
\hline \hline 1 & 1 & 2 & 3 & 4 \\
\hline 2 & 1 & 1 & 1 & 4 \\
\hline 3 & 1 & 2 & 1 & 4 \\
\hline 4 & 1 & 2 & 1 & 1 \\
\hline
\end{tabular}

Here $A$ is a JU-algebra. We find that a real valued function defined on $A$ by $\vartheta(1)=0, \vartheta(2)=\vartheta(3)=1$, and $\vartheta(4)=3$, is a pseudo-valuation on $A$.

Proposition 7. Let $\vartheta$ be a pseudo-valuation on a JU-algebras $A$. Then we have

(1) $u \leq v \Rightarrow \vartheta(v) \leq \vartheta(u)$.

(2) $\vartheta((u \diamond(v \diamond w)) \diamond w) \leq \vartheta(u)+\vartheta(v)$ for all $u, v, w \in A$.

Proof. (1) Let $u, v \in A$ be such that $u \leq v$. Replacing $u=1, v=u, w=v$ in Definition 6 and Definition 1, we get $\vartheta(v)=\vartheta(1 \diamond v) \leq \vartheta(1 \diamond(u \diamond v))+\vartheta(u)=\vartheta(1 \diamond 1)+\vartheta(u)=\vartheta(1)+\vartheta(u)=\vartheta(u)$.

(2) If we replace $u$ by $u \diamond(v \diamond w)$ in Definition 6(2), then we get

$$
\vartheta((u \diamond(v \diamond w)) \diamond w) \leq \vartheta((u \diamond(v \diamond w)) \diamond(v \diamond w))+\vartheta(v),
$$

again applying Definition 6 (2) by choosing $u=u \diamond(v \diamond w)$ and $w=v \diamond w$, we get

$$
\begin{gathered}
\vartheta((u \diamond(v \diamond w)) \diamond w) \leq v[(u \diamond(v \diamond w)) \diamond(u \diamond(v \diamond w))]+\vartheta(u)+\vartheta(v)=\vartheta(1)+\vartheta(u)+\vartheta(v) \\
\Rightarrow \vartheta((u \diamond(v \diamond w)) \diamond w) \leq \vartheta(u)+\vartheta(v) .
\end{gathered}
$$

Corollary 8. A pseudo-valuation $\vartheta$ on a JU-algebra A satisfies the inequality $\vartheta(u) \geq 0$ for all $u \in A$.

Proposition 9. If $\vartheta$ is a pseudo-valuation on a JU-algebra $A$, then we have $\vartheta((u \diamond v) \diamond v) \leq \vartheta(u)$ for all $u, v \in A$.

Proof. It is easy to see that the required inequality holds by considering $v=1$ and $w=v$ in Proposition 7 (2) and using Definition 1. 
Following results are devoted to find conditions for a real valued function on a JU-algebra $A$ to be a pseudo-valuation.

Theorem 10. Let $\vartheta$ be a real valued function on a JU-algebra A satisfying the following conditions:

(a) If $\vartheta(a) \leq \vartheta(u)$ for all $u \in A$, then $\vartheta(a)=0$,

(b) $\vartheta(u \diamond v) \leq \vartheta(v)$ for all $u, v \in A$,

(c) $\vartheta((u \diamond(v \diamond w)) \diamond w) \leq \vartheta(u)+\vartheta(v)$,

(d) $\vartheta(v \diamond(u \diamond w)) \leq \vartheta(u \diamond(v \diamond w))$.

Then $\vartheta$ is a pseudo-valuation on $A$.

Proof. From Lemma 4 and given condition (b), we have $\vartheta(1)=\vartheta(u \diamond u) \leq \vartheta(u)$ for all $u \in A$ and hence $\vartheta(1)=0$, using given condition (a). Now, from Definition 1 , Lemma 4 and given condition (c), we get $\vartheta(v)=$ $\vartheta(1 \diamond v)=\vartheta(((u \diamond v) \diamond(u \diamond v)) \diamond v) \leq \vartheta(u \diamond v)+\vartheta(u)$ for all $u, v \in A$. It follows from given condition (d) that $\vartheta(u \diamond w) \leq \vartheta(v \diamond(u \diamond w))+\vartheta(v) \leq \vartheta(u \diamond(v \diamond w))+\vartheta(v)$ for all $u, v, w \in A$. Therefore $\vartheta$ is a pseudo-valuation on $A$.

Corollary 11. Let $\vartheta$ be a real-valued function on a JU-algebra A satisfying the following conditions:

(a) $\vartheta(1)=0$,

(b) $\vartheta(u \diamond v) \leq \vartheta(v)$, for all $u, v \in A$,

(c) $\vartheta((u \diamond(v \diamond w) \diamond w)) \leq \vartheta(u)+\vartheta(v)$ for all $u, v, w \in A$,

(d) $\vartheta(v \diamond(u \diamond w)) \leq \vartheta(u \diamond(v \diamond w))$.

Then $\vartheta$ is a pseudo-valuation on $A$.

Theorem 12. If $\vartheta$ is a pseudo-valuation on a JU-algebra $A$, then $\vartheta(v) \leq \vartheta(u \diamond v)+\vartheta(u)$, for all $u, v \in A$.

Proof. Let $m=(u \diamond v) \diamond v$ for any $u, v \in A$, and $n=u \diamond v$. Then $v=1 \diamond v=(((u \diamond v) \diamond v) \diamond((u \diamond v) \diamond v)) \diamond v=$ $(m \diamond(n \diamond v)) \diamond v$. It follows from Proposition 7(2) and Proposition 9 that $\vartheta(v)=\vartheta((m \diamond(n \diamond v)) \diamond v) \leq \vartheta(m)+$ $\vartheta(n)=\vartheta((u \diamond v) \diamond v)+\vartheta(u \diamond v) \leq \vartheta(u)+\vartheta(u \diamond v)$. This completes the proof.

Theorem 13. Let $\vartheta$ be a real-valued function on a JU-algebra A satisfying the following conditions.

(1) $\vartheta(1)=0$,

(2) $\vartheta(v) \leq \vartheta(u \diamond v)+\vartheta(u)$,

(3) $\vartheta(v \diamond(u \diamond w)) \leq \vartheta(u \diamond(v \diamond w))$ for all $u, v, w \in A$.

Then $\vartheta$ is a pseudo-valuation on $A$.

Proof. For any $u, v, a, b \in A$, and using 4 with given condition (2) and (3) we get, $\vartheta(u \diamond v) \leq \vartheta(v \diamond(u \diamond v))+$ $\vartheta(v) \leq \vartheta(u \diamond(v \diamond v))+\vartheta(v)=\vartheta(v \diamond(1))+\vartheta(v)=\vartheta(1)+\vartheta(v)=\vartheta(v)$. Also,

$$
\begin{aligned}
\vartheta[(b \diamond(a \diamond u)) \diamond u] & \leq \vartheta[a \diamond((b \diamond(a \diamond u)) \diamond u)]+\vartheta(a) \\
& \leq \vartheta[(b \diamond(a \diamond u)) \diamond(a \diamond u)]+\vartheta(a) \\
& \leq \vartheta[b \diamond[(b \diamond(a \diamond u)) \diamond(a \diamond u)]+\vartheta(a)+\vartheta(b) \\
& \leq \vartheta[(b \diamond(a \diamond u)) \diamond(b \diamond(a \diamond u))]+\vartheta(a)+\vartheta(b) \\
& =\vartheta(1)+\vartheta(a)+\vartheta(b) \\
& =\vartheta(a)+\vartheta(b) .
\end{aligned}
$$

By Corollary 11, we get that $\vartheta$ is a pseudo-valuation on $A$.

Proposition 14. If $\vartheta$ is a pseudo-valuation on a JU-algebra $A$, then

$$
a \leq b \diamond u \Rightarrow \vartheta(u) \leq \vartheta(a)+\vartheta(b) \text { for all } a, b, u \in A .
$$

Proof. Suppose that $a, b, u \in A$ such that $a \leq b \diamond u$. Then by Proposition 7 (2) and Theorem 12, we have $\vartheta(u) \leq \vartheta((a \diamond(b \diamond u)) \diamond u)+\vartheta(a \diamond(b \diamond u))=\vartheta((a \diamond(b \diamond u)) \diamond u)+\vartheta(1)=\vartheta((a \diamond(b \diamond u)) \diamond u)$ $\leq \vartheta(a)+\vartheta(b)$. 
Proposition 15. Suppose that $A$ is JU-algebra. Then every pseudo-valuation $\vartheta$ on A satisfies the following inequality: $\vartheta(u \diamond w) \leq \vartheta(u \diamond v)+\vartheta(v \diamond w)$, for all $u, v, w \in A$.

Proof. It follows from $J U_{1}$ and Proposition 14.

Theorem 16. If $\vartheta$ is a pseudo-valuation on a JU-algebra $A$, then the set $I:=\{u \in A \mid \vartheta(u)=0\}$ is an ideal of $A$.

Proof. We have $\vartheta(1)=0$ and hence $1 \in I$. Next, $u, v, w \in A$ be such that $v \in I$ and $u \diamond(v \diamond w) \in I$. Then $\vartheta(v)=0$ and $\vartheta(u \diamond(v \diamond w))=0$. By Definition 6(2), we get $\vartheta(u \diamond w) \leq \vartheta(u \diamond(v \diamond w))+\vartheta(v)=0$ so that $\vartheta(u \diamond w)=0$. Hence $u \diamond w \in I$, therefore $I$ is an ideal of $A$.

Example 2. [16] Let $A=\{1,2,3,4,5\}$ in which $\diamond$ is defined by the following table

\begin{tabular}{|c||c|c|c|c|c|}
\hline$\diamond$ & 1 & 2 & 3 & 4 & 5 \\
\hline \hline 1 & 1 & 2 & 3 & 4 & 5 \\
\hline 2 & 1 & 1 & 3 & 4 & 5 \\
\hline 3 & 1 & 2 & 1 & 4 & 4 \\
\hline 4 & 1 & 1 & 3 & 1 & 3 \\
\hline 5 & 1 & 1 & 1 & 1 & 1 \\
\hline
\end{tabular}

It is easy to see that $A$ is a JU-algebra. Now, define a real-valued function $\vartheta$ on $A$ by $\vartheta(1)=\vartheta(2)=\vartheta(3)=$ $0, \vartheta(4)=3$, and $\vartheta(5)=1$. Then $I:=\{u \in A \mid \vartheta(u)=0\}=\{1,2,3\}$ is the ideal of $A$. But $\vartheta$ is not a pseudo-valuation as $\vartheta(3 \diamond 5) \not \leq \vartheta(3 \diamond(5 \diamond 5))+\vartheta(5)$.

For a real-valued function $\vartheta$ on a JU-algebra $A$, define a mapping $d_{\vartheta}: X \times X \rightarrow \mathbb{R}$ by $d_{\vartheta}(u, v)=\vartheta(u \diamond$ $v)+\vartheta(v \diamond u)$ for all $(u, v) \in A \times A$. We have following result.

Theorem 17. Let $A$ is a JU-algebra. If a real-valued function $\vartheta$ on $A$ is a pseudo-valuation on $A$, then $d_{\vartheta}$ is a pseudo-metric on $A$, and so $\left(X, d_{\vartheta}\right)$ is a pseudo-metric space. (The $d_{\vartheta}$ is called pseudo-metric induced by pseudo-valuation ७.)

Proof. Clearly, $d_{\vartheta}(u, v) \geq 1, m_{\vartheta}(u, u)=1$ and $m_{\vartheta}(u, v)=m_{\vartheta}(v, u)$ for all $u, v \in A$. For any $u, v, w \in A$ from Proposition 15, we get $d_{\vartheta}(u, v)+d_{\vartheta}(v, w)=[\vartheta(u \diamond v)+\vartheta(v \diamond u)]+[\vartheta(v \diamond w)+\vartheta(w \diamond v)]=[\vartheta(u \diamond v)+\vartheta(v \diamond$ $w)]+[\vartheta(w \diamond v)+\vartheta(v \diamond u)] \geq \vartheta(u \diamond w)+\vartheta(w \diamond u)=d_{\vartheta}(u, w)$. Hence $\left(X, d_{\vartheta}\right)$ is a pseudo-metric space.

Proposition 18. Let $A$ is a JU-algebra. Then every pseudo-metric $d_{\vartheta}$ induced by pseudo-valuation $\vartheta$ satisfies the following inequalities:

(1) $d_{\vartheta}(u, v) \geq d_{\vartheta}(x \diamond u, x \diamond v)$,

(2) $d_{\vartheta}(u \diamond v, x \diamond y) \leq d_{\vartheta}(u \diamond v, x \diamond v)+d_{\vartheta}(x \diamond v, x \diamond y)$ for all $u, v, x, y \in A$.

Proof. (1) Let $u, v, a \in A$. By $J U_{1} u \diamond v \leq(x \diamond v) \diamond(x \diamond u)$ and $v \diamond u \leq(x \diamond u) \diamond(x \diamond v)$. It follows from Proposition $7(1)$ that $\vartheta(u \diamond v) \geq \vartheta((x \diamond v) \diamond(x \diamond u))$ and $\vartheta(v \diamond u) \geq v((x \diamond u) \diamond(x \diamond v))$. So $d_{\vartheta}(u, v)=\vartheta(u \diamond v)+\vartheta(v \diamond u) \geq$ $\vartheta((x \diamond u) \diamond(x \diamond u))+\vartheta((x \diamond u) \diamond(x \diamond v))=d_{\vartheta}(x \diamond u, x \diamond v)$.

(2) Followed by definition of pseudo-metric.

Theorem 19. Let $\vartheta$ be a real-valued function on a JU-algebra $A$, if $d_{\vartheta}$ is a pseudo-metric on $A$, then $\left(X \times X, d_{\vartheta}^{\diamond}\right)$ is a pseudo-metric space, where

$$
d_{\vartheta}^{\diamond}((u, v),(a, b))=\max \left\{d_{\vartheta}(u, a), d_{\vartheta}(v, b)\right\} \text { for all }(u, v),(a, b) \in A \times A .
$$

Proof. Suppose $d_{\vartheta}$ is a pseudo-metric on $A$. For any $(u, v),(a, b) \in A \times A$, we have $d_{\vartheta}^{\diamond}((u, v),(u, v))=$ $\max \left\{d_{\vartheta}(u, u), d_{\vartheta}(v, v)\right\}=0$ and

$$
d_{\vartheta}^{\diamond}((u, v),(a, b))=\max \left\{d_{\vartheta}(u, a), d_{\vartheta}(v, b)\right\}=\max \left\{d_{\vartheta}(a, u), d_{\vartheta}(b, v)\right\}=d^{\diamond}((a, b),(u, v)) .
$$


Now let $(u, v),(a, b),(u, v) \in A \times A$. Then we have

$$
\begin{aligned}
d_{\vartheta}^{\diamond}((u, v),(u, v))+d_{\vartheta}^{\diamond}((u, v),(a, b)) & =\max \left\{d_{\vartheta}(u, u), d_{\vartheta}(v, v)\right\}+\max \left\{d_{\vartheta}(u, a), d_{\vartheta}(v, b)\right\} \\
& \geq \max \left\{d_{\vartheta}(u, u)+d_{\vartheta}(u, a), d_{\vartheta}(v, v)+d_{\vartheta}(v, b)\right\} \\
& \geq \max \left\{d_{\vartheta}(u, a), d_{\vartheta}(v, b)\right\}=d_{\vartheta}^{\diamond}((u, v),(a, b)) .
\end{aligned}
$$

Hence $\left(X \times X, d_{\vartheta}^{\diamond}\right)$ is a pseudo-metric space.

Corollary 20. If $\vartheta: X \rightarrow \mathbb{R}$ is a pseudo-valuation on a JU-algebra $A$, then $\left(X \times X, d_{\vartheta}^{\diamond}\right)$ is a pseudo-metric space.

Theorem 21. Let $A$ is a JU-algebra. If $\vartheta: X \rightarrow \mathbb{R}$ is a valuation on $A$, then $\left(X, d_{\vartheta}\right)$ is a metric space.

Proof. Suppose $\vartheta$ is a valuation on $A$, then $\left(X, d_{\vartheta}\right)$ is a pseudo-metric space by Theorem 19. Further consider $u, v \in A$ be such that $d_{\vartheta}(u, v)=0$, then $0=d_{\vartheta}(u, v)=\vartheta(u \diamond v)+\vartheta(v \diamond u)$, and hence $\vartheta(u \diamond v)=0$ and $\vartheta(v \diamond u)=0$ since $\vartheta(u) \geq 0$ for all $u \in A$ and, since $\vartheta$ is a valuation on $A$, it follows that $u \diamond v=1$ and $v \diamond u=1$ so from (condition in the given theorem) that $u=v$. Hence $\left(X, d_{\vartheta}\right)$ is a metric space.

Theorem 22. Let $A$ is a JU-algebra. If $\vartheta: X \rightarrow \mathbb{R}$ is a valuation on $A$, then $\left(X \times X, d_{\vartheta}^{\diamond}\right)$ is a metric space.

Proof. From Corollary 20, we know that $\left(X \times X, d_{\vartheta}^{\diamond}\right)$ is a pseudo-metric space. Suppose $(u, v),(a, b) \in A \times A$ be such that $d_{\vartheta}^{\diamond}((u, v),(a, b))=0$, then $0=d_{\vartheta}^{\diamond}((u, v),(a, b))=\max \left\{d_{\vartheta}(u, a), d_{\vartheta}(v, b)\right\}$, and so $d_{\vartheta}(u, a)=0=$ $d_{\vartheta}(v, b)$. Since $d_{\vartheta}(u, v) \geq 0$ for all $(u, v) \in A \times A$. Hence $0=d_{\vartheta}(u, a)=\vartheta(u \diamond a)+\vartheta(a \diamond u)$ and $0=d_{\vartheta}(v, b)=$ $\vartheta(v \diamond b)+\vartheta(b \diamond v)$. It follows that $\vartheta(u \diamond a)=0=\vartheta(a \diamond u)$ and $\vartheta(v \diamond b)=0=v(b \diamond v)$ so that $u \diamond a=1=a \diamond u$ and $v \diamond b=0=b \diamond v$. Now we have $a=u$ and $b=v$, and so $(u, v)=(a, b)$, therefore $\left(X \times X, d_{\vartheta}^{\diamond}\right)$ is a metric space.

Theorem 23. Let $A$ is a JU-algebra. If $\vartheta$ is a valuation on $A$, then the operation $\diamond$ in $A$ is uniformly continuous.

Proof. Consider for any $\epsilon>0$, if $d_{\vartheta}^{\diamond}((u, v),(a, b))<\frac{\epsilon}{2}$ then $d_{\vartheta}(u, a)<\frac{\epsilon}{2}$ and $d_{\vartheta}(v, b)<\frac{\epsilon}{2}$. This implies that $d_{\vartheta}(u \diamond v, a \diamond b) \leq d_{\vartheta}(u \diamond v, a \diamond v)+d_{\vartheta}(a \diamond v, a \diamond b) \leq d_{\vartheta}(u, a)+d_{\vartheta}(v, b)<\frac{\epsilon}{2}+\frac{\epsilon}{2}=\epsilon$ (from Proposition 18). Therefore the operation $\diamond: X \times X \rightarrow A$ is uniformly continuous.

Author Contributions: All authors contributed equally to the writing of this paper. All authors read and approved the final manuscript.

Conflicts of Interest: "The authors declare no conflict of interest."

\section{References}

[1] Buşneag, C. (2007). Valuations on residuated lattices. Annals of the University of Craiova-Mathematics and Computer Science Series, 34, 21-28.

[2] Buşneag, D. (2003). On extensions of pseudo-valuations on Hilbert algebras. Discrete Mathematics, 263(1-3), 11-24.

[3] Buşneag D. (1996). Hilbert algebras with valuations. Mathematica Japonica, 44(2), 285-289.

[4] Doh, M. I., \& Kang, M. S. (2010). BCK/BCI-algebras with pseudo-valuations. Honam Mathematical Journal, 32(2), 217-226.

[5] Ghorbani, S. (2010). Quotient BCI-algebras induced by pseudo-valuations. Iranian Journal of Mathematical Sciences and Informatics, 5(2), 13-24.

[6] Zhan, J., \& Jun, Y. B. (2013). (Implicative) Pseudo-Valuations On $R_{0}$-Algebras. University Politehnica Of Bucharest Scientific Bulletin-Series A-Applied Mathematics And Physics, 75(4), 101-112.

[7] Yang, Y., \& Xin, X. (2017). EQ-algebras with pseudo pre-valuations. Italian Journal of Pure and Applied Maths, 36, $29-48$.

[8] Mehrshad, S., \& Kouhestani, N. (2018). On Pseudo-Valuations on BCK-Algebras. Filomat, 32(12), 4319-4332.

[9] Jun, Y. B., Ahn, S. S., \& Roh, E. H. (2012). BCC-algebras with pseudo-valuations. Filomat, 26(2), $243-252$.

[10] Iampan, A. (2017). A new branch of the logical algebra: UP-algebras. Journal of Algebra and Related Topics, 5(1), 35-54.

[11] Yaqoob, N., Mostafa, S. M., \& Ansari, M. A. (2013). On cubic KU-ideals of KU-algebras. ISRN Algebra, 2013.

[12] Ansari, M. A., \& Koam, A. N. (2018). Rough approximations in KU-algebras. Italian Journal of Pure and Applied Mathematics, 40, 679-691.

[13] Ansari, M. A., Koam, A. N., \& Haider, A. (2019). Rough set theory applied to UP-algebras. Italian Journal of Pure and Applied Mathematics, 42. 388-402. 
[14] Ansari, M., Haidar, A., \& Koam, A. (2018). On a Graph Associated to UP-Algebras. Mathematical and Computational Applications, 23(4), 61.

[15] Romanoa, D. A. (2019). Pseudo-Valuations on UP-Algebras. Universal Journal of Mathematics and Applications, 2(3), 138-140.

[16] Ansari, M. A., Haider, A., \& Koam, A. N. (2020). On JU-algebras and p-Closure Ideals. Computer Science, 15(1), 135-154.

[17] Kawila, K., Udomsetchai, C., \& Iampan, A. (2018). Bipolar fuzzy UP-algebras. Mathematical and Computational Applications, 23(4), 69.

(C) 2019 by the authors; licensee PSRP, Lahore, Pakistan. This article is an open access article distributed under the terms and conditions of the Creative Commons Attribution (CC-BY) license (http://creativecommons.org/licenses/by/4.0/). 